\title{
BMJ Open ANalgesic Efficacy and safety of MOrphiNe versus methoxyflurane in patients with acute myocardial infarction: the rationale and design of the ANEMON-SIRIO 3 study: a multicentre, open-label, phase II, randomised clinical trial
}

\author{
Aldona Kubica, ${ }^{1}$ Agata Kosobucka (D) , ${ }^{1}$ Piotr Niezgoda, ${ }^{2}$ Piotr Adamski, ${ }^{2}$ \\ Katarzyna Buszko, ${ }^{3}$ Maciej Lesiak, ${ }^{4}$ Wojciech Wojakowski, ${ }^{5}$ Mariusz Gasior, ${ }^{6}$ \\ Jarosław Goracy, ${ }^{7}$ Andrzej Kleinrok, ${ }^{8,9}$ Klaudiusz Nadolny, ${ }^{10,11}$ Eliano Navarese, ${ }^{2}$ \\ Jacek Kubica ${ }^{2}$
}

To cite: Kubica A, Kosobucka A, Niezgoda P, et al. ANalgesic Efficacy and safety of MOrphiNe versus methoxyflurane in patients with acute myocardial infarction: the rationale and design of the ANEMON-SIRIO 3 study: a multicentre, openlabel, phase II, randomised clinical trial. BMJ Open 2021;11:e043330. doi:10.1136/ bmjopen-2020-043330

- Prepublication history for this paper is available online. To view these files, please visit the journal online (http://dx.doi. org/10.1136/bmjopen-2020043330).

Received 13 August 2020 Revised 10 December 2020 Accepted 25 January 2021

Check for updates

(c) Author(s) (or their employer(s)) 2021. Re-use permitted under CC BY-NC. No commercial re-use. See rights and permissions. Published by BMJ.

For numbered affiliations see end of article.

Correspondence to

Dr Agata Kosobucka akosobucka@wp.pl

\section{ABSTRACT}

Introduction The unfavourable influence of morphine on the pharmacokinetics of ticagrelor resulting in weaker and retarded antiplatelet effect in patients with acute coronary syndrome (ACS) has been previously shown. Replacing morphine with methoxyflurane, a potent, non-opioid analgesic agent, that does not weaken or delay the effect of antiplatelet agents may improve the clinical efficacy of treatment of patients with ACS.

Methods The ANEMON-SIRIO 3 study was designed as a multicentre, open-label, phase II, randomised clinical trial aimed to test the analgesic efficacy and safety of methoxyflurane in patients with ACS. The study population will comprise patients with ST-elevation myocardial infarction or non-ST-elevation ACS admitted to the study centres with typical chest pain requiring analgesic treatment. Before percutaneous coronary intervention (PCl) for the patients with index ACS will be randomly assigned in 1:1 ratio to receive methoxyflurane administered by inhalation, or to obtain morphine administered intravenously. Analgesic treatment will be followed by $300 \mathrm{mg}$ loading dose of aspirin and $180 \mathrm{mg}$ loading dose of ticagrelor. Patients will be assessed with regard to pain intensity according to the Numeric Pain Rating Scale at baseline, 3 min after study drug administration and immediately after PCI. Moreover, patients will be actively monitored with regard to the occurrence of side effects of evaluated therapies, as well as adverse events that may be related to insufficient platelet inhibition (no-reflow phenomenon assessed immediately after PCl, administration of GPIlb/llla inhibitors during PCl, acute stent thrombosis).

Ethics and dissemination The study will be conducted in six Polish clinical centres from the beginning of in accordance with the ethical standards of the institutional research committee and with the 1964 Helsinki

\section{Strengths and limitations of this study}

- The ANEMON-SIRIO 3 study is designed as a multicentre, open-label, phase II, randomised clinical trial.

- This is the first study aimed to test analgesic efficacy and safety of methoxyflurane in patients with acute coronary syndrome.

- The trial is designed to compare methoxyflurane with morphine which is current analgesic standard of care for chest pain in acute coronary syndromes.

- The study is not powered for the evaluation of Major Adverse Cardiovascular Events (MACE).

- The open label design of the study is burdened with possible evaluation bias of analgesic effect of compared agents.

declaration and its later amendments or comparable ethical standards.

Trial registration details ClinicalTrials.gov, NCT04476173.

\section{INTRODUCTION}

Platelet activation plays a pivotal role in the pathophysiology of acute coronary syndromes (ACS). Pharmacological platelet inhibition with P2Y12 receptor antagonists and aspirin, together with percutaneous coronary intervention (PCI) are the cornerstone of treatment of patients with ACS. ${ }^{1-8}$

Chest pain and anxiety are both associated with sympathetic activation, which increases workload of the heart. Relieving of these symptoms in acute myocardial infarction 
(AMI) is expected to improve the balance between the demand for oxygen and its supply. Morphine, apart from its analgesic effects, also alleviates the work of breathing and reduces anxiety. However, despite its favourable analgesic and sedative actions, morphine also exerts adverse effects, which include vomiting and reduction of gastrointestinal motility. These side effects affect the intestinal absorption of oral drugs co-administered with morphine. ${ }^{9}{ }^{10}$ Previously performed randomised studies revealed unfavourable influence of morphine on the pharmacokinetics of ticagrelor resulting in weaker and retarded antiplatelet effect. ${ }^{11-16}$ Similar effect was observed for clopidogrel ${ }^{17} 18$ and prasugrel, ${ }^{17} 1920$ but not for aspirin. ${ }^{9}$ Unfortunately, different tested strategies failed to overcome the 'morphine effect'. ${ }^{21-23}$ Therefore, replacing morphine with another highly effective non-opioid analgesic that does not weaken and does not delay the effect of antiplatelet agents may improve the clinical efficacy of treatment of patients with AMI. An alternative analgesic treatment strategy in patients with ST-elevation myocardial infarction (STEMI) treated with ticagrelor was tested in the ON-TIME 3 trial. Intravenous acetaminophen in comparison with intravenous fentanyl was equally effective in pain relief, however expected difference in platelet reactivity was not significant despite significantly higher ticagrelor plasma concentrations in the intravenous acetaminophen arm. ${ }^{24}$

Methoxyflurane was shown to be effective and well tolerated for the management of acute traumatic pain with a rapid onset of analgesia. This volatile, non-opioid analgesic agent is self-administered with a hand-held inhaler. ${ }^{25} 26$ As it does not affect the $\mu$-opioid receptors, which inhibit propulsive motility and secretion of the gastrointestinal tract, methoxyflurane is not expected to decrease or delay absorption or effects of orally administered drugs, including P2Y12 inhibitors, as well as to exert any other negative impact in patients with ACS.

However analgesic efficacy and safety of methoxyflurane have never been tested in this clinical setting. The concept to apply methoxyflurane in patients with ACS is supported by recently published results of the AVOID study. ${ }^{27}$ The reported incidence of no-reflow phenomenon after PCI in patients with STEMI was higher in the high-dose compared with low-dose opioid group. Moreover, higher doses of opioids were associated with greater infarct size. ${ }^{27}$

\section{METHODS}

\section{Study design and population}

The ANEMON-SIRIO 3 study was designed as a multicentre, open-label, phase II, randomised clinical trial. Six Polish clinical centres are expected to participate in the trial. The enrolment to the study is scheduled from 1 September 2020 to 31 May 2021. The study population will comprise patients consecutively admitted to the study centres due to ACS with typical chest pain requiring analgesic treatment. Patients aged from 18 to 80 years with STEMI or very high risk non-ST-elevation ACS (NSTE-ACS) with recurrent/ refractory chest pain requiring immediate invasive treatment are eligible to participate in the study. The diagnosis of STEMI and non-STEMI will be made according to the Fourth Universal Definition of Myocardial Infarction, ${ }^{28}$ and unstable angina (UA) will be diagnosed according to the European Society of Cardiology (ESC) guidelines for the management of NSTE-ACS. ${ }^{29}$ The following exclusion criteria were defined: any previously administered analgesic medication for the index ACS, pregnancy, manifest infection or inflammatory state, cardiogenic shock during screening for eligibility, respiratory failure, heart failure (NYHA (New York Heart Association) class III or IV during screening for eligibility), uncontrolled hypertension (systolic blood pressure $>180 \mathrm{~mm} \mathrm{Hg}$ or diastolic blood pressure $>100 \mathrm{~mm} \mathrm{Hg}$ ).

\section{Ethics and dissemination}

The study will be conducted in accordance with the principles contained in the Declaration of Helsinki. The ANEMON-SIRIO 3 study protocol and the template informed consent forms received approval from the Bioethics Committee (BC) of the Collegium Medicum, Nicolaus Copernicus University in Torun (study approval reference number KB 38/2020). The investigators will report the progress and safety of the study to the $\mathrm{BC}$ and to the Data Safety and Monitoring Board every 2 months. The final report (manuscript for publication) of the study results will prepared within 1 month after study completion. The report will be available at ClinicalTrials.gov Identifier: NCT04476173.

\section{Treatment protocol and concomitant medications}

Patients with initial diagnosis of STEMI or NSTE-ACS will be screened for eligibility for the study. The informed consent will be obtained in the setting of acute pain therefore the abbreviated information regarding the study and investigated treatment will be provided verbally. The patient will receive full written information, and will sign the consent with the abbreviated and full version of the information. Before PCI for the index ACS, after obtaining informed consent patients will be enrolled and randomly assigned with a secure online system in 1:1 ratio to one of two study arms. The study design is depicted in figure 1 .

Patients in the intervention arm will receive methoxyflurane administered by inhalation ( $3 \mathrm{mg}$ vial), whereas those in the control arm will obtain $5 \mathrm{mg}$ of morphine administered intravenously. Efficacy of analgesics in both arms will be evaluated $3 \mathrm{~min}$ after initiation of treatment (study endpoint). The Numeric Pain Rating Scale (NPRS) will be applied for pain intensity assessment. Improvement is defined as reduction of pain intensity by at least 2 NPRS points. The pathways of analgesic treatment in both arms are shown on figure 2A,B. According to the study protocol, unbearable chest pain persisting regardless of the administered analgesics and performed coronary revascularisation, will be considered a strong indication for a crossover, which will be left to the discretion of the investigator. 


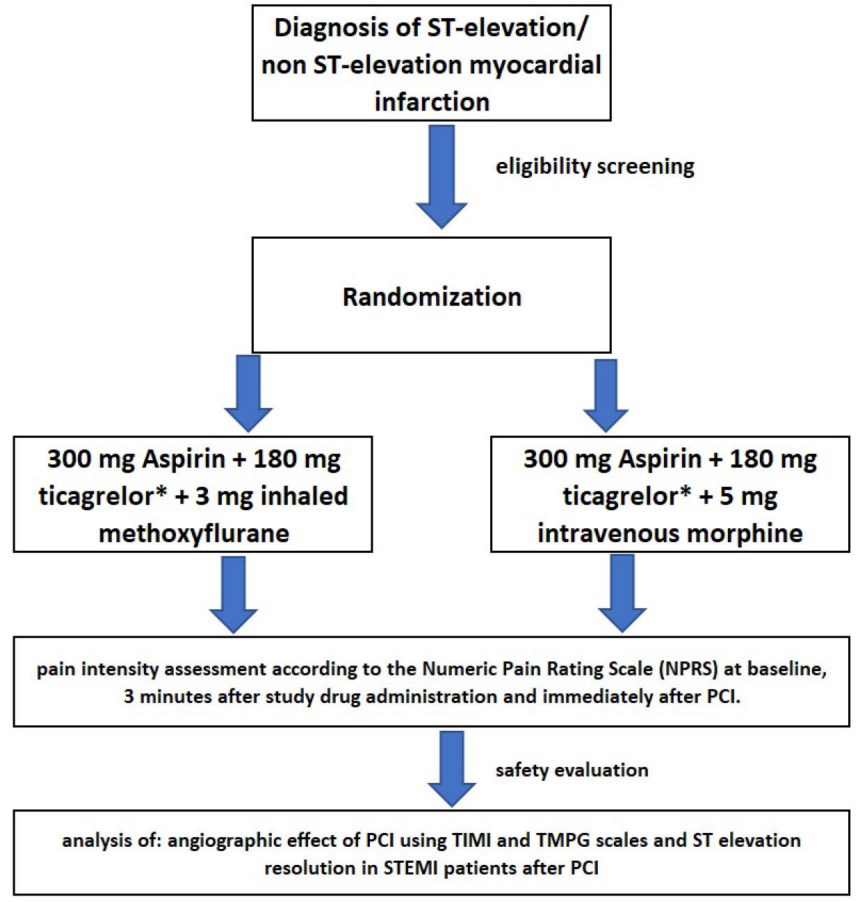

Figure 1 The study design. *Patients previously loaded with clopidogrel will be reloaded with ticagrelor after randomisation. $\mathrm{PCl}$, percutaneous coronary intervention; STEMI, ST-elevationmyocardial infarction; TIMI, Thrombolysis In Myocardial Infarction; TMPG, TIMI Myocardial Perfusion Grade.

Analgesic treatment in all enrolled subjects will be followed by $300 \mathrm{mg}$ loading dose of aspirin and $180 \mathrm{mg}$ loading dose of ticagrelor. Subjects loaded with clopidogrel or prasugrel before the study enrolment will be re-loaded with $180 \mathrm{mg}$ ticagrelor in line with the European Society of Cardiology (ESC) recommendations. ${ }^{7}$ All patients during their participation in the study, will be treated according to the ESC guidelines, including primary PCI. ${ }^{78}$ Patients will be observed up to 24 hours after PCI. During their participation in the study, all patients will be assessed with regard to pain intensity according to the NPRS at baseline, 3 min after study drug administration and immediately after PCI. Moreover, patients will be actively monitored with regard to the occurrence of side effects of evaluated therapies, as well as adverse events that may be related to insufficient platelet inhibition (no-reflow phenomenon assessed immediately after PCI, administration of GPIIb/IIIa inhibitors during PCI, acute stent thrombosis). Platelet reactivity will be assessed with VerifyNow System 2 hours after loading with P2Y12 receptor inhibitor. Clinical research showed that nephrotoxicity was a dose-related complication caused by fluoride ions produced by O-demethylation of methoxyflurane. It was observed at high anaesthetic doses, but not reported with low analgesic doses. ${ }^{30}$ Therefore, renal function will not be assessed before enrolment into the study. Nevertheless, renal function

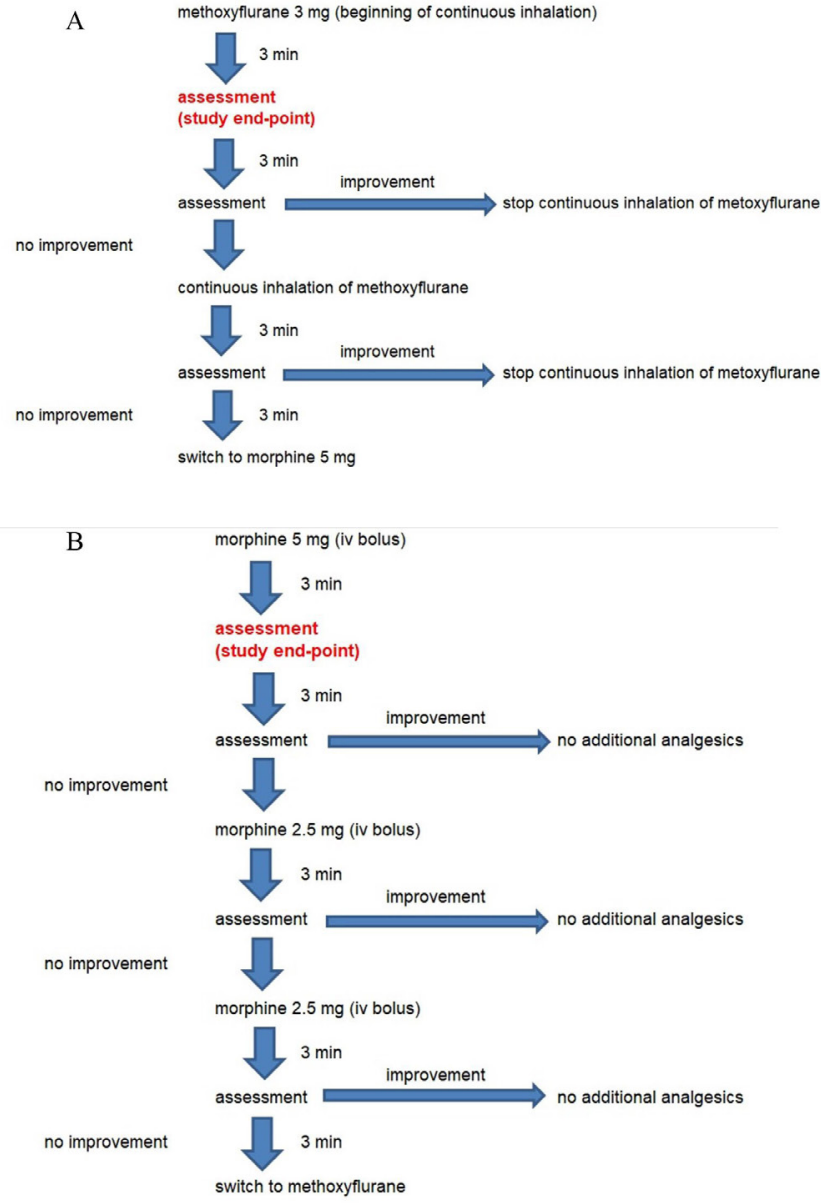

Figure 2 (A) The pathway of analgesic treatment in methoxyflurane arm. (B) The pathway of analgesic treatment in morphine arm. iv, intravenous.

will be monitored after coronary intervention due to possible contrast-induced kidney injury. ${ }^{31}$

\section{Study endpoints}

Two co-primary endpoints are defined as the measure of pain intensity according to the NPRS 3 min after drug administration and immediately after PCI in relation to pain intensity assessed before drug administration.

The following secondary endpoints are established: adverse effects of evaluated therapies (nausea, vomiting, dry mouth, respiratory failure - need for intubation, headache, dizziness, drowsiness, loss of consciousness, death), need for GPIIb/IIIa inhibitor administration during PCI due to large intracoronary thrombus. Additionally, a central analysis of: (1) angiographic effect of PCI using TIMI and TMPG scales and (2) ST elevation resolution in patients with STEMI after PCI (with a 70\% resolution cut-off) will be done.

\section{Determination of sample size}

We assume that inclusion of 200 patients (1:1 ratio between intervention and control arms) would be sufficient to show non-inferiority of methoxyflurane as compared with morphine with regard to analgesic effect in patients with ACS with a two-sided alpha value of 0.05 and at least $80 \%$ power. Since there is no reference study, we decided to 
perform an internal pilot phase of the study including the first 100 patients (50 patients for each arm) for estimating the final sample size.

\section{Patient and public involvement \\ No patient involved.}

\section{Statistical analysis}

The continuous variables in the two arms will be compared with parametric tests (Student's t-test) or non-parametric tests (Mann-Whitney test), if the assumptions for parametric analysis are not satisfied. The categorical data will be analysed with $\chi^{2}$ test and the Fisher's exact test. Eventfree survival analysis will be performed by the KaplanMeier method with log-rank test for group comparison. Tests results yielding $\mathrm{p}<0.05$ will be considered as statistically significant.

\section{DISCUSSION}

The antiplatelet drugs administration is of vast importance, especially in the initial phase of ACS treatment. ${ }^{3432}$ Results of the PLATO trial have shown that the therapy with ticagrelor, a novel potent platelet P2Y12 receptor inhibitor, reduces all-cause mortality and the rates of cardiovascular events compared with clopidogrel in patients with ACS, including STEMI. ${ }^{5}$ The observed superiority of ticagrelor over clopidogrel in decreasing ischaemic event rate in patients with ACS has been attributed to a fast, potent and uniform pharmacodynamic features. ${ }^{6}$ The ECS recommends ticagrelor (180 mg loading dose, followed by $90 \mathrm{mg}$ two times per day) on top of aspirin in patients with ACS regardless of initial treatment strategy. ${ }^{78}$ The same guidelines recommend use of titrated opioid agents, (eg, morphine) as a treatment of choice for pain relief in this clinical setting. ${ }^{8}$ The highly possible impact of morphine-ticagrelor interaction on clinical outcomes in patients with AMI has never been proven. ${ }^{10}$ The negative effect of drug-drug interaction can possibly be balanced by an analgesic effect of morphine. Therefore, replacing morphine with another highly effective non-opioid analgesic that does not weaken and does not delay the effect of antiplatelet agents may improve the clinical efficacy of treatment of patients with ACS.

\section{Study limitations}

It should be highlighted that the ANEMON-SIRIO 3 study is not powered for the evaluation of Major Adverse Cardiovascular Events (MACE) or angiographic endpoints. Moreover, the open label design of the study is burdened with possible evaluation bias of analgesic effect of compared agents.

In summary, the aim of the ANEMON-SIRIO 3 study is to evaluate analgesic efficacy and safety of treatment with inhaled methoxyflurane versus intravenously administered morphine for pain relief in patients with ACS treated with PCI.
Author affiliations

${ }^{1}$ Departament of Health Promotion, Nicolaus Copernicus University in Toruń Ludwik Rydygier Collegium Medicum, Bydgoszcz, Poland

${ }^{2}$ Department of Cardiology and Internal Medicine, Nicolaus Copernicus University in Toruń Ludwik Rydygier Collegium Medicum, Bydgoszcz, Poland

${ }^{3}$ Department of Theoretical Foundations of Biomedical Science and Medical Informatics, Nicolaus Copernicus University in Toruń Ludwik Rydygier Collegium Medicum, Bydgoszcz, Poland

${ }^{4}$ The 1st Department of Cardiology, Poznan University of Medical Sciences, Poznan, Poland

${ }^{5}$ Department of Cardiology and Structural Heart Diseases, Medical University of Silesia, Katowice, Poland

${ }^{6} 3$ rd Department of Cardiology, School of Medicine with the Division of Dentistry, Medical University of Silesia, Zabrze, Poland

${ }^{7}$ Department of Cardiology, Pomeranian Medical University in Szczecin, Szczecin, Poland

${ }^{8}$ Department of Cardiology, Pope John Paul II Reginal Hospital, Zamosc, Poland

${ }^{9}$ Medical Department, University of Information Technology and Management, Rzeszow, Poland

${ }^{10}$ Department of Emergency Medicine and Disasters, Medical University of Bialystok, Bialystok, Poland

${ }^{11}$ Department of Emergency Medical Service, Higher School of Strategic Planning, Dąbrowa Górnicza, Poland

Contributors AKu: Conceived the idea for the study, contributed to the design of the research, drafted the article, gave approval of final version of the manuscript. AKo: Contributed to the design of the research, edited the manuscript, gave approval of final version of the manuscript. PN: Contributed to the design of the research, gave approval of final version of the manuscript. PA: Revised the manuscript, gave approval of final version of the manuscript. KB: Drafted the article - statistical part, gave approval of final version of the manuscript. ML, WW, MG, JG, AKI, KN: Edited the manuscript, gave approval of final version of the manuscript. EN: Revised the manuscript, gave approval of final version of the manuscript. JK: Contributed to the design of the research, drafted the article, gave approval of final version of the manuscript.

Funding This research received financial support from the Nicolaus Copernicus University, Poland, through IDUB Project (Emerging Field - Metabolic Civilisation Diseases, NCU-EF/LS1/1/2019).

Competing interests AKu, PA, ML, WW, MG, JG, KN, EN, JK: fee for lectures and involvement in AstraZeneca advisory board.

Patient and public involvement Patients and/or the public were not involved in the design, or conduct, or reporting, or dissemination plans of this research.

Patient consent for publication Not required.

Provenance and peer review Not commissioned; externally peer reviewed.

Open access This is an open access article distributed in accordance with the Creative Commons Attribution Non Commercial (CC BY-NC 4.0) license, which permits others to distribute, remix, adapt, build upon this work non-commercially, and license their derivative works on different terms, provided the original work is properly cited, appropriate credit is given, any changes made indicated, and the use is non-commercial. See: http://creativecommons.org/licenses/by-nc/4.0/.

ORCID iD

Agata Kosobucka http://orcid.org/0000-0002-1368-3475

\section{REFERENCES}

1 Gurbel PA, Myat A, Kubica J. State of the art: oral antiplatelet therapy. JRSM Cardiovasc Dis 2016;5:204800401665251.

2 Myat A, Tantry US, Kubica J, et al. Current controversies in the use of aspirin and ticagrelor for the treatment of thrombotic events. Expert Rev Cardiovasc Ther 2016;14:1361-70.

3 Kubica J, Adamski P, Paciorek P, et al. Treatment of patients with acute coronary syndrome: recommendations for medical emergency teams: focus on antiplatelet therapies. updated experts' standpoint. Cardiol J 2018;25:291-300.

4 Kubica J, Adamski P, Paciorek P, et al. Anti-aggregation therapy in patients with acute coronary syndrome - recommendations for medical emergency teams. Experts' standpoint. Kardiol Pol 2017;75:399-408. 
5 Wallentin L, Becker RC, Budaj A, et al. Ticagrelor versus clopidogrel in patients with acute coronary syndromes. $N$ Engl J Med 2009;361:1045-57.

6 Adamski P, Adamska U, Ostrowska M, et al. Evaluating current and emerging antithrombotic therapy currently available for the treatment of acute coronary syndrome in geriatric populations. Expert Opin Pharmacother 2018;19:1415-25.

7 Valgimigli M, Bueno H, Byrne RA, et al. 2017 ESC focused update on dual antiplatelet therapy in coronary artery disease developed in collaboration with EACTS: the task force for dual antiplatelet therapy in coronary artery disease of the European Society of cardiology (ESC) and of the European association for Cardio-Thoracic surgery (EACTS). Eur Heart J 2018;39:213-60.

8 Ibanez B, James S, Agewall S, et al. 2017 ESC guidelines for the management of acute myocardial infarction in patients presenting with ST-segment elevation: the task force for the management of acute myocardial infarction in patients presenting with ST-segment elevation of the European Society of cardiology (ESC). Eur Heart $J$ 2018;39:119-77.

9 Bartko J, Schoergenhofer C, Schwameis M, et al. Morphine interaction with aspirin: a double-blind, crossover trial in healthy volunteers. J Pharmacol Exp Ther 2018;365:430-6.

10 Kubica J, Kubica A, Jilma B, et al. Impact of morphine on antiplatelet effects of oral P2Y12 receptor inhibitors. Int $J$ Cardiol 2016;215:201-8.

11 Kubica J, Adamski P, Ostrowska M, et al. Influence of morphine on pharmacokinetics and pharmacodynamics of ticagrelor in patients with acute myocardial infarction (impression): study protocol for a randomized controlled trial. Trials 2015;16:198.

12 Kubica J, Adamski P, Ostrowska M, et al. Morphine delays and attenuates ticagrelor exposure and action in patients with myocardial infarction: the randomized, double-blind, placebo-controlled IMPRESSION trial. Eur Heart $J$ 2016;37:245-52.

13 Kubica J, Kubica A, Jilma B, et al. Impact of morphine on antiplatelet effects of oral P2Y12 receptor inhibitors. Int $J$ Cardiol 2016;215:201-8.

14 Adamski P, Ostrowska M, Sikora J, et al. Comparison of ticagrelor pharmacokinetics and pharmacodynamics in STEMI and NSTEMI patients (PINPOINT): protocol for a prospective, observational, single-centre study. BMJ Open 2017;7:e013218.

15 Adamski P, Sikora J, Laskowska E, et al. Comparison of bioavailability and antiplatelet action of ticagrelor in patients with ST-elevation myocardial infarction and non-ST-elevation myocardial infarction: a prospective, observational, single-centre study. PLOS One 2017;12:e0186013.

16 Adamski P, Buszko K, Sikora J, et al. Determinants of high platelet reactivity in patients with acute coronary syndromes treated with ticagrelor. Sci Rep 2019;9:3924.

17 Vaidya GN, Khan A, Ghafghazi S. Effect of morphine use on oral P2Y12 platelet inhibitors in acute myocardial infarction: metaanalysis. Indian Heart J 2019;71:126-35.

18 Hobl E-L, Stimpfl T, Ebner J, et al. Morphine decreases clopidogrel concentrations and effects: a randomized, double-blind, placebocontrolled trial. J Am Coll Cardiol 2014;63:630-5.
19 Hobl E-L, Reiter B, Schoergenhofer C, et al. Morphine interaction with prasugrel: a double-blind, cross-over trial in healthy volunteers. Clin Res Cardiol 2016;105:349-55.

20 Siller-Matula JM, Specht S, Kubica J, et al. Abciximab as a bridging strategy to overcome morphine-prasugrel interaction in STEMI patients. Br J Clin Pharmacol 2016;82:1343-50.

21 Sikora J, Niezgoda P, Barańska M, et al. Metoclopramide administration as a strategy to overcome MORPHine-ticagrelOr interaction in patients with unstable angina PectorlS-The metamorphosis trial. Thromb Haemost 2018;118:2126-33.

22 Niezgoda P, Sikora J, Barańska M, et al. Crushed sublingual versus oral ticagrelor administration strategies in patients with unstable angina. A pharmacokinetic/pharmacodynamic study. Thromb Haemost 2017;117:718-26.

23 Niezgoda P, Barańska MA, Sikora J, et al. Oral naloxone to overcome the moRphine effect in acute COronary syndrome patients treated with ticagrelor - NARCOTIC trial. Cardiol J 2020 doi:10.5603/ CJ.a2020.0040

24 Tavenier AH, Hermanides RS, Ottervanger JP, et al. Impact of opioids on P2Y12-receptor inhibition in patients with STelevation myocardial infarction who are pre-treated with crushed ticagrelor: opioids aNd crushed ticagrelor in myocardial infarction evaluation (ON-TIME 3) trial. Eur Heart J Cardiovasc Pharmacother 2020;30:pvaa095.

25 Blair HA, Frampton JE. Methoxyflurane: a review in trauma pain. Clin Drug Investig 2016;36:1067-73.

26 Coffey F, Dissmann P, Mirza K, et al. Methoxyflurane analgesia in adult patients in the emergency department: a subgroup analysis of a randomized, double-blind, placebo-controlled study (STOP!). Adv Ther 2016;33:2012-31.

27 Fernando H, Nehme Z, Peter K, et al. Prehospital opioid dose and myocardial injury in patients with ST elevation myocardial infarction. Open Heart 2020;7:e001307.

28 Thygesen K, Alpert JS, Jaffe AS, et al. Fourth universal definition of myocardial infarction (2018). Circulation 2018;138:e618-51.

29 Roffi M, Patrono C, Collet J-P, et al. 2015 ESC guidelines for the management of acute coronary syndromes in patients presenting without persistent ST-segment elevation: Task force for the management of acute coronary syndromes in patients presenting without persistent ST-segment elevation of the European Society of cardiology (ESC). Eur Heart J 2016;37:267-315.

30 Porter KM, Dayan AD, Dickerson S, et al. The role of inhaled methoxyflurane in acute pain management. Open Access Emerg Med 2018;10:149-64.

31 Pyka Łukasz, Hawranek M, Wilczek K, et al. Imaging-guided percutaneous coronary intervention with ultra-low contrast angiographic control for patients at extreme risk of contrast induced nephropathy. Cardiol J 2019;26:796-8.

32 Zhang K, Yang W, Zhang M. Pretreatment with antiplatelet drugs improves the cardiac function after myocardial infarction without reperfusion in a mouse model. Cardiol J 2019 doi:10.5603/ CJ.a2019.0051 